\title{
High-performance silicon scanning mirror for laser printing
}

\author{
Wyatt O. Davis ${ }^{* a}$, Dean Brown ${ }^{\mathrm{a}}$, Mark Helsel ${ }^{\mathrm{b}}$, Randy Sprague ${ }^{\mathrm{a}}$, Greg Gibson ${ }^{\mathrm{a}}$, Arda Yalcinkaya ${ }^{\mathrm{c}}$, \\ Hakan Urey ${ }^{\mathrm{c}}$ \\ ${ }^{\text {a }}$ Microvision, Inc., $6222185^{\text {th }}$ Avenue NE, Redmond, WA, USA 98052 \\ ${ }^{\mathrm{b}}$ Dept. of Nanotechnology, North Seattle Community College, 9600 College Way North, Seattle, \\ WA, USA 98103-3599 \\ ${ }^{\mathrm{c} C o l l e g e ~ o f ~ E n g i n e e r i n g, ~ K o c ̧ ~ U n i v e r s i t y, ~ R u m e l i ~ F e n e r i ~ Y o l u, ~ S a r i y e r ~ 34450, ~ I s t a n b u l, ~ T u r k e y ~}$
}

\begin{abstract}
This paper describes the design, fabrication, and characterization of the first MEMS scanning mirror with performance matching the polygon mirrors currently used for high-speed consumer laser printing. It has reflector dimensions of $8 \mathrm{~mm}$ $\mathrm{X} 0.75 \mathrm{~mm}$, and achieves $80^{\circ}$ total optical scan angle at an oscillation frequency of $5 \mathrm{kHz}$. This performance enables the placement of approximately 14,000 individually resolvable dots per line at a rate of 10,000 lines per second, a recordsetting speed and resolution combination for a MEMS scanner. The scanning mirror is formed in a simple microfabrication process by gold reflector deposition and patterning, and through-wafer deep reactive-ion etching. The scanner is actuated by off-the-shelf piezo-ceramic stacks mounted to the silicon structure in a steel package. Device characteristics predicted by a mathematical model are compared to measurements.
\end{abstract}

Keywords: Laser printer, MEMS, scanning mirror, piezoelectric actuator

\section{INTRODUCTION}

A typical laser printing system uses a spinning polygon mirror to scan a focused and modulated laser spot onto a photoconductive drum, which in turn picks up toner by electrostatic attraction and transfers it to paper [1,2]. High-speed laser printers for consumer and office use currently deliver 600-2400 dot-per-inch (dpi) resolution at a printing speed of 30-40 pages-per-minute ( $\mathrm{ppm}$ ). In this paper, we describe a one-axis micromachined silicon scanning mirror designed to deliver 1200 dpi resolution at $40 \mathrm{ppm}$, showing the capacity of a MEMS scanning mirror to deliver performance competitive with the incumbent polygon mirror based approach.

The resolution and scan speed requirements for laser printing are well beyond the capabilities of most scanning technologies using MEMS, galvonometers, or mechanical-resonant scanning mirrors. Printing at 1200 dpi resolution requires the formation of a $22 \mu \mathrm{m}$ FWHM laser spot on the photoconductive drum. This establishes a minimum scanning mirror reflector size: for this work the minimum size is $8 \mathrm{~mm}$ in the same direction as the scan, and $0.75 \mathrm{~mm}$ perpendicular to the scan. A total optical scan angle (TOSA) of $80^{\circ}$ is required to print to A4-sized paper, and a scan frequency of $5 \mathrm{kHz}$ produces a line rate of 10,000 lines per second ( 2 lines per scan period), which translates to $40 \mathrm{ppm}$.

Resolution and scan frequency are useful bases of comparison for scanning mirrors' performance. The maximum achievable resolution (for displays, the number of distinguishable pixels or for printers, the distinguishable dpi) is proportional to $\theta^{*} D$, where $\theta$ is the maximum scan angle and $D$ is the mirror dimension that influences the spot size at a focal point [3]. The $\theta^{*} D$ achieved in this work is $640^{\circ}(\mathrm{TOSA})^{*} \mathrm{~mm}$. The scanning frequency $f$ is also relevant because it determines the rate of information transmission, for example the refresh rate for displays or the page feed rate for printers. Microvision, Inc. [4] and Fraunhofer Institute for Photonic Microsystems (IPMS) [5] produce MEMS scanning mirrors for commercial high-resolution scanned laser beam displays and imaging systems, but the mirror sizes and/or scan angles of these and other existing devices are not sufficient for printing applications, as shown by the chart in Figure 1. There are vast numbers of other scanning mirrors not shown in the chart, however it is often unclear from published information if the dynamic mirror deformation while scanning at large angles and high frequencies is small

\footnotetext{
*wyatt_davis@microvision.com, phone 425.882.6718, fax 425.936.4660, microvision.com
} 
enough to permit the formation of a compact focused spot. Maintaining sufficient mirror flatness is one of the primary challenges to scanning with a large mirror to large angles at high frequencies.

\section{Scanning Mirror Performance Comparison}

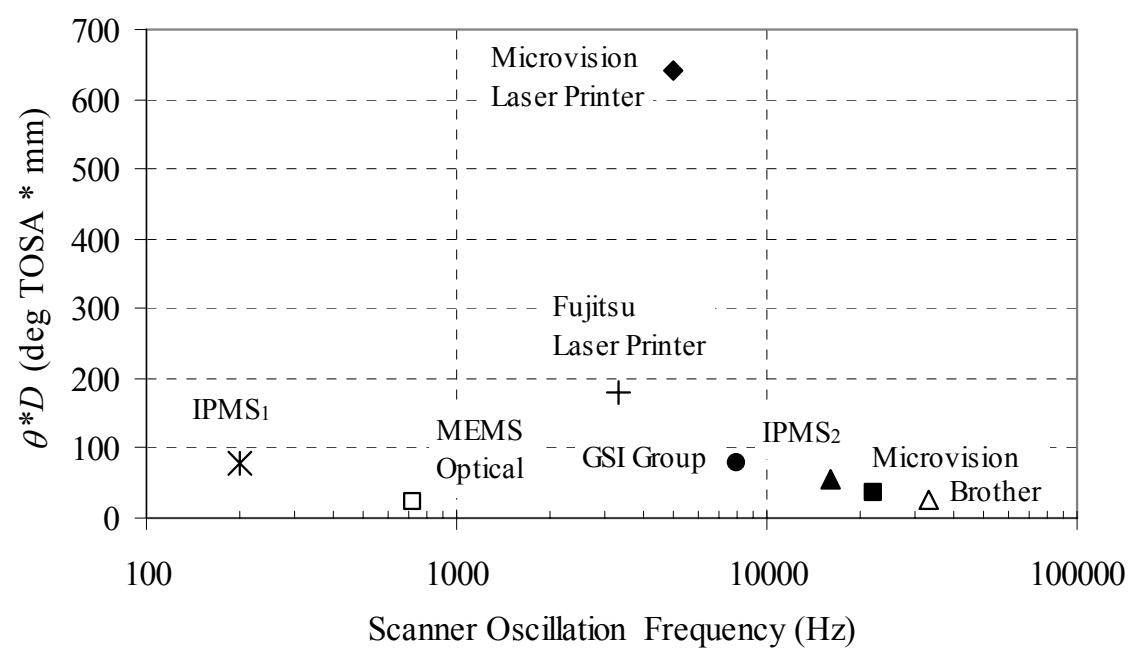

Figure 1 Comparison of silicon laser printer scanning mirror performance to several other previously reported scanning mirrors. All but the GSI Group Inc. CRS scanning mirror device [6] are micromachined. Five of the scanning mirrors in the chart are for 1-D scanning (the Microvision Inc. laser printer (this work), IPMS ${ }_{1}$ for barcode scanning [7], the GSI Group Inc. scanning mirror, Fujitsu Laboratories, Ltd. laser printer mirror [8], and Brother Industries, Ltd. display scanning mirror [9]). The scanning mirror from MEMS Optical Inc. [10] is for 2-D pointing/scanning, while all of the others operate at resonance and so are for scanning only. The scanning mirror labeled IPMS $_{2}$ [11] is a $0.5 \mathrm{~mm} 2-\mathrm{D}$ scanning mirror capable of large scan angles and recently used for medical imaging [12], and the Microvision Inc. scanning mirror is deployed in SVGA resolution scanned laser display systems [13].

\section{SCANNING MIRROR DESIGN}

\subsection{Mechanical design}

For sinusoidal motion, the scanning mirror requirements of $8 \mathrm{~mm}$ dimension, $80^{\circ} \mathrm{TOSA}$, and $5 \mathrm{kHz}$ scan frequency imply a maximum speed of $44 \mathrm{~m} / \mathrm{sec}$ at the tip of the mirror, and a peak acceleration of $140 \mathrm{kG}$. Keeping the mirror dynamically flat to within $1 / 4 \lambda$ peak-to-valley $(\mathrm{P}-\mathrm{V})$ under such large inertial loads presents a challenge. The use of single-crystal silicon as the mirror material is well-motivated. It has a strength-to-weight ratio superior to most materials [14], which addresses the issue of dynamic flatness. Furthermore, it is generally not susceptible to fatigue failure, and it is compatible with well-established commercially available batch microfabrication manufacturing processes [14]. The following section describes the design of a silicon scanning mirror meeting the requirements for laser printing described above.

The scanning mirror, with reflector dimensions of $8 \mathrm{~mm} \mathrm{X} 0.75 \mathrm{~mm}$, is suspended by torsion bars whose geometry is designed to give a resonant frequency of $5 \mathrm{kHz}$ and peak stress lower than $1 \mathrm{GPa}$ at $20^{\circ}$ mechanical scan angle (MSA). The basic geometry is illustrated in Figure 2(a). The scanning mirror operates resonantly to minimize the demands on the actuation source. The mirror and torsion bars are single-crystal silicon with a structural layer thickness of $700 \mu \mathrm{m}$. This large thickness is necessary to provide sufficient dynamic flatness for the mirror, using considerations described in [3]. Then the width and length of the torsion bars are designed to meet the stiffness and peak stress requirements. The mirror flatness, resonant frequency, and stress considerations are confirmed with finite element analysis using ANSYS ${ }$, for example Figure 2(b) shows the first principal stress in the torsion bars at peak scan angle. The maximum stress occurs in the middle of the sidewall faces. 


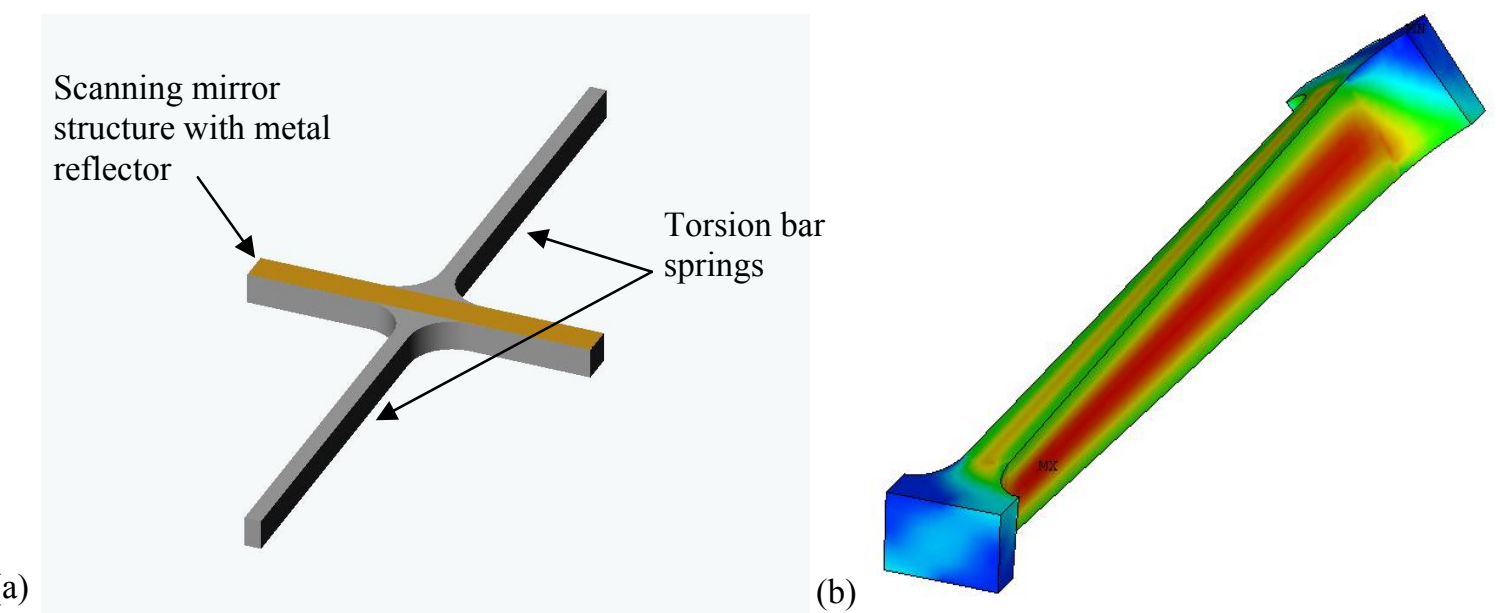

Figure 2 (a) Conceptual illustration of the scanning mirror structure consisting of a reflector patterned on a block of silicon and suspended by comparatively flexible torsion bar springs. (b) First principal stress contours showing maximum stress occurring on the torsion bar sidewalls when the scanning mirror is rotated to peak angle.

For a resonant mechanical system undergoing steady-state oscillations, the applied excitation must overcome the energy dissipation due to damping. Other forces and torques intrinsic to the oscillator such as inertial loads and restoring forces due to mechanical stiffness are exactly balanced. For a torsional oscillator, the required torque amplitude $T_{0}$ to achieve scan angle amplitude $\theta_{0}$ at resonance is

$$
T_{0}=\theta_{0} \omega_{0} c=\frac{K \theta_{0}}{Q},
$$

where $\omega_{0}$ is the resonant frequency, $c$ is a linear damping coefficient, $K$ is the spring constant, and $Q$ is the quality factor. In this case the scan angle and resonant frequency are design targets, but knowledge of the damping coefficient is less certain and its value is typically difficult to influence. Therefore, estimating the damping (or $Q$ ) can be the most important consideration in determining actuator requirements.

The air flow mechanics for the oscillating mirror can be classified by estimating the Reynolds number Re,

$$
\mathrm{Re}=\frac{W V_{\max }}{v},
$$

where $W$ is a characteristic length, for example the mirror's minor dimension of $750 \mu \mathrm{m}, V_{\max }$ is a characteristic velocity taken as the maximum speed of the mirror scanning at its desired operating conditions, and $v$ is the kinematic viscosity of air at standard temperature and pressure. This calculation gives $\mathrm{Re}=2200$ at $20^{\circ} \mathrm{MSA}$, indicating that the flow induced by the scanning mirror is likely to be turbulent.

The estimate of the damping torque $T_{d}$ on the oscillating scanning mirror is derived by computing the form drag on a differential area on the mirror surface, computing its torque and integrating over the surface,

$$
T_{d}=-2 \int_{0}^{D / 2} \frac{1}{2} c_{D} \rho W l^{3} \dot{\theta}^{2} \operatorname{Sign}(\dot{\theta}) d l=-\frac{1}{64} c_{D} \rho W D^{4} \dot{\theta}^{2} \operatorname{Sign}(\dot{\theta})=-c_{q} \dot{\theta}^{2} \operatorname{Sign}(\dot{\theta})
$$

where $c_{D}$ is a drag coefficient, which has an estimated upper bound of $c_{D}=2$ for $\operatorname{Re}>1$ [15], and $c_{q}$ is a quadratic damping coefficient.

This damping torque estimate predicts a quality factor $Q$ of 1640 for the scanning mirror operating at $20^{\circ}$ MSA. This estimate is used to evaluate options for scanning mirror actuation. 


\subsection{Actuator selection}

Options available for generating the excitation torque required to overcome the damping include electromagnetic, electrostatic, and piezoelectric actuation. Microvision, Inc. currently produces MEMS scanning mirrors for displays that use electromagnetic actuation with a movable coil suspended in a magnetic field. However, for the printer application, the number of coil turns required on the printer scanning mirror was computed to be impractically large, resulting in excessively high resistive power dissipation and a large silicon structure. Another electromagnetic actuation scheme uses the torque generated on a permanent magnet or permeable material attached to the scanning mirror by the variable field generated by an external electromagnet $[16,17]$. This approach incurs the significant penalty of the attached magnet's inertia oscillating at the scanning frequency. This quickly leads to large overall silicon dimensions because of the stiffer torsion springs required to maintain $5 \mathrm{kHz}$ resonance while staying within design limits for stress.

Electrostatic actuation is commonly used for MEMS, including scanning mirrors [7, 8, 11, 18-20], with the two basic configurations being gap-closing and comb-drive. The large displacements required of the printer scanning mirror do not easily permit small gaps for either configuration, and so the actuation voltages would be unreasonably high. Likewise, piezoelectric actuation is impractical for directly applying torque to the scanning mirror because it is generally restricted to small displacement applications.

For resonant systems, actuator demands can often be simplified by applying a displacement excitation to the base of the suspension springs that support the body. This principle is used effectively in Microvision's magnetically actuated scanning mirror to generate the large angle, high frequency scanning motion for the horizontal axis of a display [13]. In this way, an actuator design problem requiring both high forces and large displacements can be recast as a problem requiring much smaller displacements. Electromagnetic and electrostatic actuator options are available for a base excitation scheme for the printer scanning mirror, however, the size of the scanning mirror in Figure 2 and the need to keep the fabrication and assembly processes simple make actuation with piezoelectric stack actuators highly attractive. Integrating the actuators with the mounting of the torsion bar ends to a housing results in a simple overall construction requiring no additional microfabrication or adhesive bonding. Piezoelectric ceramic materials have proven effective for MEMS actuation in diverse applications, e.g. [9, 21], and are readily available from several suppliers.

Figure 3 shows a conceptual diagram for the scanning mirror and actuation system. The main torsion springs terminate at comparatively stiff tee structures, or T-bars, with mounting pads at the ends. The mounting pads sit on top of the piezo stack actuators, so that the T-bars undergo bending deformations under linear displacements of the actuators. For actuation frequencies well-below resonance, the main torsion springs deform very little, and the mirror rotates to an angle commensurate with the slope of the center of the T-bar. When the frequency is swept higher through the resonant frequency of the scanning mirror, mechanical amplification occurs. At resonance the mirror will achieve an angle of $Q$ times the angle at the base, where $Q$ is the quality factor that depends on the damping.

A lumped-parameter mathematical model for the coupled dynamics of the scanning mirror, T-bar structure, and piezo actuators was developed using mathematical analysis and the results of finite element analysis. Using the damping estimate described above, the model predicts that the piezo actuators will be required to apply $45.6 \mathrm{~N}$ of force and displace the T-bar ends by $+/-0.4 \mu \mathrm{m}$. For this work, the piezo stack actuators selected were Physik Instrumente PICMA ${ }^{\circledR} 885.10$, and the lumped parameters in the mathematical model are available from the data sheet [22]. The actuator has dimensions $5 \mathrm{~mm}$ X 5mm X 10mm, with an unblocked displacement limit of $8 \mu \mathrm{m}$ at $120 \mathrm{~V}$ and a blocking force of $800 \mathrm{~N}$. The estimated force and displacement requirements are well within the capabilities of this piezo actuator, with substantial overhead to allow for errors in the estimates of damping and other parameters. The estimated voltage amplitude required to drive the resonating scanning mirror to $20^{\circ} \mathrm{MSA}$ is $14.6 \mathrm{~V}$ peak-to-peak with a corresponding $2 \mathrm{~W}$ peak required to drive the piezo actuators' $0.6 \mu \mathrm{F}$ capacitance. 


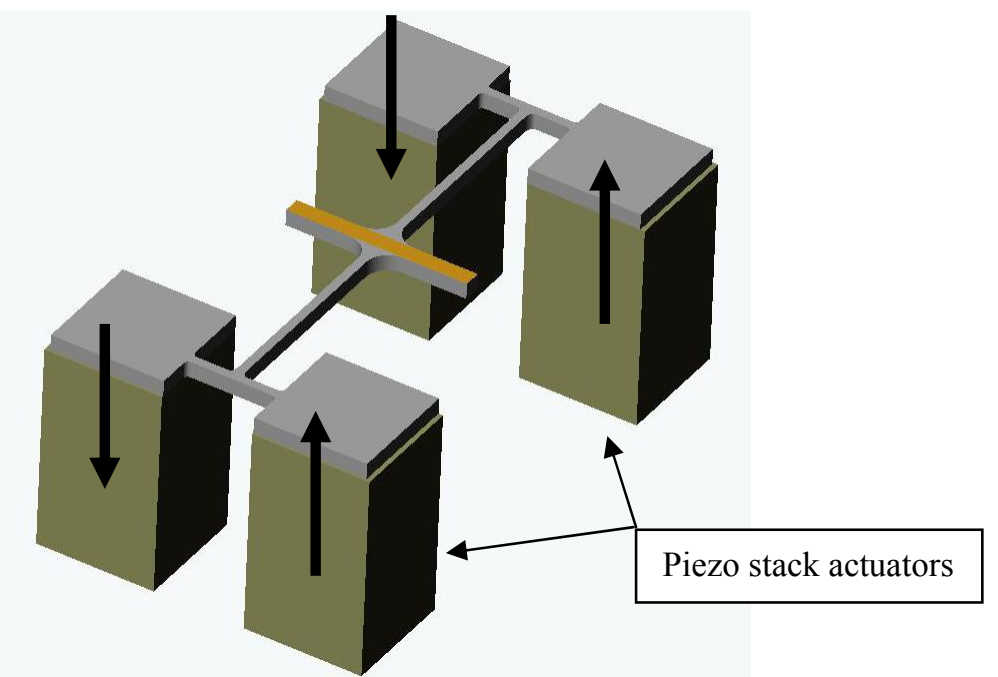

Figure 3 Conceptual model for piezoelectric stack actuator excitation of the torsion bar spring bases. The arrows indicate the direction of expansion or contraction of the actuators $z$ inducing scanning mirror rotation $\theta$.

\section{SCANNING MIRROR FABRICATION}

Cross sections for the main steps in the microfabrication process for forming silicon scanning mirrors are shown in Figure 4. A gold metal layer forming the reflector is deposited and patterned on a polished $700 \mu \mathrm{m}$ thick silicon wafer. Through-wafer DRIE using a thick photoresist mask forms the scanning mirror. The shape of the scanning mirror permits a close-packed layout of devices, but this precludes standard dicing procedures. To achieve device singulation, narrow silicon beams connect neighboring scanning mirrors at the mounting areas that form the mechanical interface with the piezo actuators. Breaking these fragile beams separates the scanning mirrors.

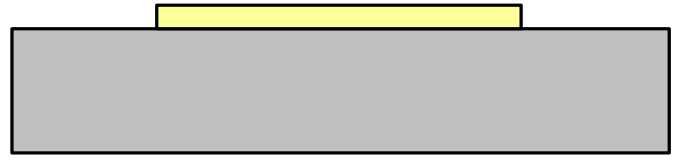

\section{(a)}

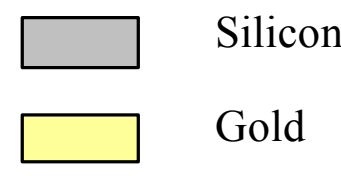

(b)

Figure 4 Process flow cross sections for scanning mirror fabrication: (a) Deposit and pattern gold for reflector. (b) Through-wafer DRIE etch.

\section{EXPERIMENTAL MEASUREMENTS}

The prototypes assembled and tested for this work used set screws and load spreading pressure plates to clamp the scanning mirror's mounting pads to the piezo actuators, with all of the components mounted in steel housings measuring approximately $30 \mathrm{~mm}$ X $32 \mathrm{~mm}$ X $28 \mathrm{~mm}$. 


\subsection{Optical characteristics}

Typical measured reflectivity of the gold layer on silicon is $90 \%-93 \%$ at $650 \mathrm{~nm}$. The peak-to-valley (P-V) mirror deformation measured with an interferometer on six assembled scanning mirror systems ranged from $0.04 \lambda$ to $0.18 \lambda$, indicating very flat reflective surfaces within acceptable limits.

Figure 5 shows the wavefront distortion due to dynamic mirror deformation at $20^{\circ} \mathrm{MSA}$, where inertial loads that deform the mirror are at a maximum, as measured by a Shack-Hartmann wavefront sensor using strobed illumination. The method is described in [23]. The dynamic mirror deformation measured on six assembled scanning mirrors ranged from $0.14 \lambda$ to $0.27 \lambda$, confirming the oscillating mirror is a suitable optical surface for use in laser printing.

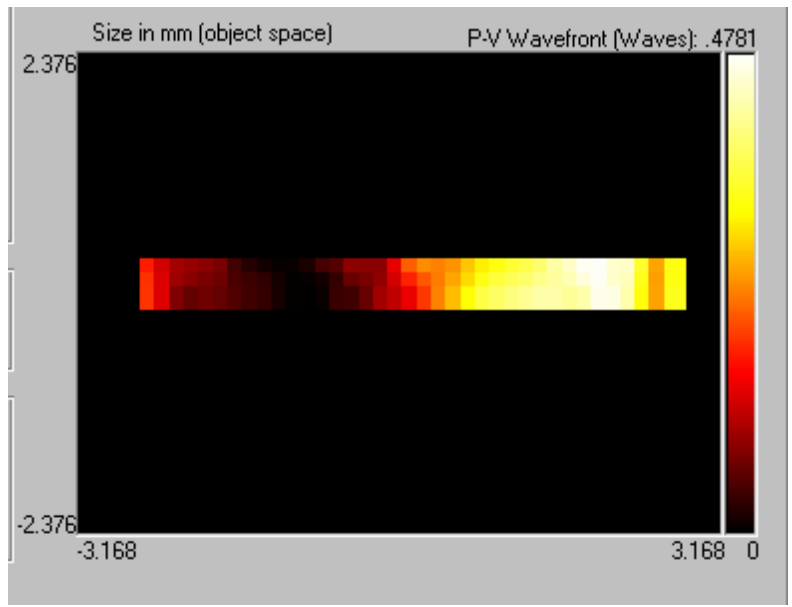

Figure 5 Wavefront distortion from the reflective surface of the scanning mirror operating at $20^{\circ} \mathrm{MSA}$ measured by a ShackHartmann wavefront sensor.

\subsection{Scanning mirror performance}

The average quality factor $Q$ for 10 tested prototypes was 1196 at $20^{\circ}$ MSA (computed using the half-power formula [24] and the frequency response), $38 \%$ lower than the predicted value of 1640 . The average piezo drive voltage required was $24.7 \mathrm{~V}$ peak-to-peak, $69 \%$ higher than the predicted value of $14.6 \mathrm{~V}$ peak-to-peak. Part of this discrepancy is due to the overestimate of $Q$, and the rest is due to uncertainty in some of the lumped parameters used in the mathematical model due to boundary condition assumptions in the finite element model. The reactive power supply to the 4-piezo scanning mirror system is approximately $5.7 \mathrm{~W}$ peak. In spite of the discrepancies the actual drive levels required are still well within the capabilities of the chosen piezo actuators.

\section{CONCLUSIONS}

A silicon scanning mirror satisfying the challenging specifications of high-speed laser printing has been demonstrated. The performance in terms of the combination of achievable resolution (equivalently, dpi) and scanning frequency is the highest yet reported for a MEMS-based scanning mirror system. The scanning mirror assemblies are built using a simple attachment to commercially available piezoelectric stack actuators. A scan angle of $80^{\circ}$ TOSA at $5 \mathrm{kHz}$ scanning mirror oscillation frequency $(10 \mathrm{kHz}$ line scan frequency) is achieved with $24.7 \mathrm{~V}$ peak-to-peak and $5.7 \mathrm{~W}$ peak. The measured drive levels are somewhat higher than the predictions of a mathematical model, due partially to the difficulty in accurately estimating the energy losses due to air damping. 


\section{ACKNOWLEDGEMENT}

The authors wish to thank Bin Xue of Microvision, Inc. for assistance with Shack-Hartmann wavefront distortion measurements, and Brian Cleaver of Microvision, Inc. for his contributions to scanning mirror fabrication.

\section{REFERENCES}

1. Canon, Inc., www.canon.com/technology/canon_tech/category/lbp.html

2. J. M. Fleischer, M. R. Latta, M. E. Rabedeau, "Laser optical system of the IBM 3800 printer," IBM Journal of Research and Development, Sep. 1977, pp. 479-483.

3. H. Urey, D. W. Wine, and T. D. Osborn, "Optical performance requirements for MEMS-scanner based microdisplays,"

4. Microvision, Inc., www.microvision.com.

5. Fraunhofer Institut Photonische Mikrosysteme, www.ipms.fraunhofer.de.

6. GSI Group Inc., CRS Series, product brochures, www.gsig.com.

7. Fraunhofer Institut Photonische Mikrosysteme, product brochure, www.ipms.fraunhofer.de/en/products/MSD/msme.pdf

8. O. Tsuboi, X. Mi, N. Kouma, H. Okuda, H. Soneda, S. Ueda, and Y. Ikai, "A full-time accelerated vertical combdriven micromirror for high speed 45-degree scanning," MST News, No. 3, June 2004, pp. 12-14.

9. N. Asai, R. Matsuda, M. Watanabe, H. Takayama, S. Yamada, A. Mase, M. Shikida, K. Sato, M. Lebedev, J. Akedo, "A novel high resolution optical scanner actuated by aerosol deposited PZT films," IEEE MEMS 2003, Kyoto Japan, Jan. 2003.

10. MEMS Optical, Inc., product brochures, www.memsoptical.com.

11. C. Drabe, T. Klose, A. Wolter, H. Schenk, and R. James, "A new microlaser camera," Proc. SPIE vol. 6466, MOEMS and Miniaturized Systems VI, Jan. 2007

12. R. James, G. Gibson, W. O. Davis, F. Metting, and C. Drabe, "Update on MEMS-based scanned-beam imager," Proc. SPIE vol. 6466, MOEMS and Miniaturized Systems VI, Jan. 2007.

13. A. D. Yalcinkaya, H. Urey, D. Brown, T. Montague, and R. Sprague, "Two-axis electromagnetic microscanner for high resolution displays," JMEMS, v. 15, No. 4, August, 2006, pp. 786-794.

14. K. E. Petersen, "Silicon as a mechanical material," Proc. IEEE, vol. 70, No. 5, 1982.

15. J. A. Roberson and C. T. Crowe, Engineering Fluid Mechanics, Houghton Mifflin Co., 1990.

16. D.A. Horsley, W.O. Davis, K.J. Hogan, M.R. Hart, E.C. Ying, M. Chaparala, B. Behin, M.J. Daneman, "Optical and mechanical performance of a novel magnetically actuated MEMS-based optical switch," JMEMS,

17. J. W. Judy, N. Myung, "Magnetic materials for MEMS," MRS Workshop on MEMS Materials, San Francisco, CA, April 5-6, 2002.

18. S. Kwon, V. Milanovic, L. P. Lee, "High aspect ratio 2D gimbaled microscanner with large static rotation," IEEE/LEOS Optical MEMS 2002, Lugano, Switzerland, August, 2002.

19. J.-H. Lee, Y.-C. Ko, H.-M. Jeong, B.- S. Choi, J.-M. Kim, D. Y. Jeon, "SOI-based fabrication process of the scanning mirror having vertical comb fingers," Sensors and Actuators A, v. 102, 2002, pp. 11-18.

20. D. W. Wine, M. P. Helsel, L. Jenkins, H. Urey, T. D. Osborn, "Performance of a biaxial MEMS-based scanner for microdisplay applications," Proc. SPIE vol. 4178, MOEMS and Miniaturized Systems, August, 2000.

21. S.-G. Kim, K.-H. Hwang, "Thin-film micromirror array (TMA) for information display systems," Euro Display '99, Berlin, Germany, September 1999.

22. Physik Instrumente L. P., PICMA®885.10 data sheet, www.physikinstrumente.com.

23. M. Brown, T. Gong, D. R. Neal, J. Roller, S. Luanava, H. Urey, "Measurement of the dynamic deformation of a high frequency scanning mirror using a Shack-Hartmann wavefront sensor," Proc. SPIE vol. 4451, Optical Manufacturing and Testing IV, 2001.

24. S. S. Rao, Mechanical Vibrations, Addison-Wesley, 1995. 